\title{
IMMUNOCHEMICAL STUDIES ON A LAMINARIBIOSYL-AZOPROTEIN CONJUGATE*
}

\author{
P. Z. ALLEN \\ Department of Microbiology, University of Rochester \\ School of Medicine and Dentistry, Rochester, N.Y. 14620, U.S.A. \\ I. J. GOLDSTEIN \\ Department of Biological Chemistry, University of Michigan, \\ Ann Arbor, Mich. 48105, U.S.A. \\ and \\ R. N. IYER \\ Department of Pediatrics, Medical Center, Case-Western \\ Reserve University, Cleveland, Ohio 44106, U.S.A.
}

(Received 12 January 1970)

\begin{abstract}
Laminaribiose (3-o- $\beta$-D-glucopyranosyl-D-glucose) was coupled to bovine serum albumin (BSA) by an azophenyl linkage to provide the synthetic antigen BSAp-phenylazo- $\beta$-laminaribioside. The behavior of antisera prepared in rabbits immunized with the $\beta$-laminaribiosyl conjugate was examined by immunodiffusion, quantitative precipitation and hapten inhibition. Anticonjugate absorbed with carrier protein showed the greatest reactivity with the homologous $\beta$-laminaribiosyl-BSA antigen, but also showed some cross precipitation with $\beta$-cellobiosyl, $\beta$-sophorosyl and $\beta$-gentiobiosylBSA conjugates. Glucobioses linked through the $\beta-(1 \rightarrow 2), \beta-(1 \rightarrow 3), \beta-(1 \rightarrow 4)$ and $\beta$ - $(1 \rightarrow 6)$ positions, as well as laminaridextrins and tri and tetrasaccharides of $\beta$-linked glucose possessing a laminaribiose moiety either at a nonreducing end location or at a subterminal location, were assayed for their ability to inhibit antilaminaribioside precipitation. Hapten inhibition data showed anticonjugate to possess a high degree of specificity directed against the terminal nonreducing $\beta$-laminaribiosyl end group.
\end{abstract}

Artificial polyglycosylated conjugates consisting of mono, di- or trisaccharide haptenic groups coupled to protein or polypeptide carriers have been synthesized and used as antigens in several immunological studies [1-12]. The examination of sugar conjugate-anticonjugate model systems has provided immunochemical data on: (1) the relationship of carbohydrate structure to anticarbohydrate specificity $[2,4-6,9,11-16]$; (2) the estimation of the minimal size of $\alpha$-linked glucose haptenic groups required to induce antibodies showing cross reactivity with dextran [9]; (3) size of the combining region and heterogeneity of antibodies formed in response to sugar haptens $[6,9,15]$; (4) the valence and affinity of rabbit and horse anti- $\beta$-lactoside antibodies [17-23]; and (5) the effect of stereochemical and configuration alterations on the affinity of hapten-anticarbohydrate interaction [17].

Among the structural features possessed by disaccharide haptens known to contribute to immunochemical specificity is the position or point of attachment

*Supported by Research Grant GB 4578 from the National Science Foundation and by USPHS Research Grant No. AM-10171 from the National Institutes of Health. 
of the interglycosidic linkage. The position and configuration of this linkage may be systematically varied to provide a family of closely related compounds which differ from one another in molecular configuration. The contribution or role of the position of attachment of the interglycosidic linkage to immunochemical specificity and reactivity of antisugar conjugates has been only partially explored with a few systems employing $\beta$-linked diglucose conjugates as antigen. Thus far, only $\beta$-linked glucobioses linked through the $\beta$-(l $\rightarrow 6)$, the $\beta$ - $(1 \rightarrow 4)$, or the $\beta-(1 \rightarrow 2)$ position have been conjugated to protein to provide antigens for immunochemical study $[2,4,11,13]$.

In the present study, laminaribiose, the $\beta$ - $(1 \rightarrow 3)$-linked glucobiose was coupled to bovine serum albumin (BSA) to provide the synthetic antigen BSAp-phenylazo- $\beta$-laminaribioside. Rabbits immunized with the $\beta$-laminaribiosyl conjugate provided antisera used to examine the specificity and cross reactivity of anticonjugate. The behavior of rabbit anti-laminaribioside with synthetic sugar-azoprotein conjugates possessing $\beta$-laminaribiosyl, $\beta$-sophorosyl, $\beta$ cellobiosyl, $\beta$-gentiobiosyl, $\alpha$ or $\beta$-glucosyl, $\beta$-galactosyl, $\beta$-maltosyl or $\beta$-panosyl groupings was studied by immunodiffusion and quantitative precipitation.

By use of quantitative hapten inhibition, the isomeric series of glucobioses linked through the $\beta-(1 \rightarrow 2), \beta-(1 \rightarrow 3), \beta-(1 \rightarrow 4)$ and $\beta-(1 \rightarrow 6)$ positions were assayed for their ability to inhibit conjugate-anticonjugate precipitation. Assay of glucotrioses and glucotraoses with mixed linkages, possessing a laminaribiose group, either at a terminal, nonreducing location or at a subterminal location, permitted a direct evaluation of the relative reactivity of internal versus terminal nonreducing laminaribiosyl end groups with anti-laminaribioside. Immunochemical data established: (1) a specificity for anticonjugate directed against the introduced $\beta$-laminaribiosyl grouping; (2) the relative importance of nonreducing $\beta$-laminaribiosyl end groups for interaction with antibody; (3) the ability of $\beta$-cellobiosyl, $\beta$-gentiobiosyl and $\beta$-sophorosyl-azoprotein conjugates to cross precipitate fractions of anti-laminaribioside.

\section{MATERIALS AND METHODS}

\section{Glycosyl-phenylazoprotein conjugates and polysaccharides}

p-Aminophenyl glycosides of laminaribioise, cellobiose, gentiobiose, sophorose, glucose, galactose, maltose and panose were diazotized and coupled to bovine serum albumin (BSA), employing the conditions described by Westphal and Feier [24]. The carbohydrate content of conjugates estimated by the anthrone method was used to calculate the average number of sugar residues introduced per BSA molecule (Table 1). The immunochemical behavior of several of the conjugates listed, as well as their interaction with concanavalin A, has been previously described $[11-13,25,26]$. Water-soluble laminaran was purchased from Koch-Light Laboratories Ltd., Colnbrook, England, while oat glucan was a gift from Dr. A. S. Perlin.

Antisera. Four rabbits were immunized with the $\beta$-laminaribiosyl phenylazo bovine serum albumin conjugate incorporated into complete Freund's adjuvant (Difco Laboratories). The conjugate possessed $17 \cdot 7$ laminaribiosyl residues per BSA molecule. A total of $1.4 \mathrm{ml}$ of emulsion containing $1 \mathrm{mg}$ conjugate per $\mathrm{ml}$ was administered to each animal over the course of three weeks as multiple 


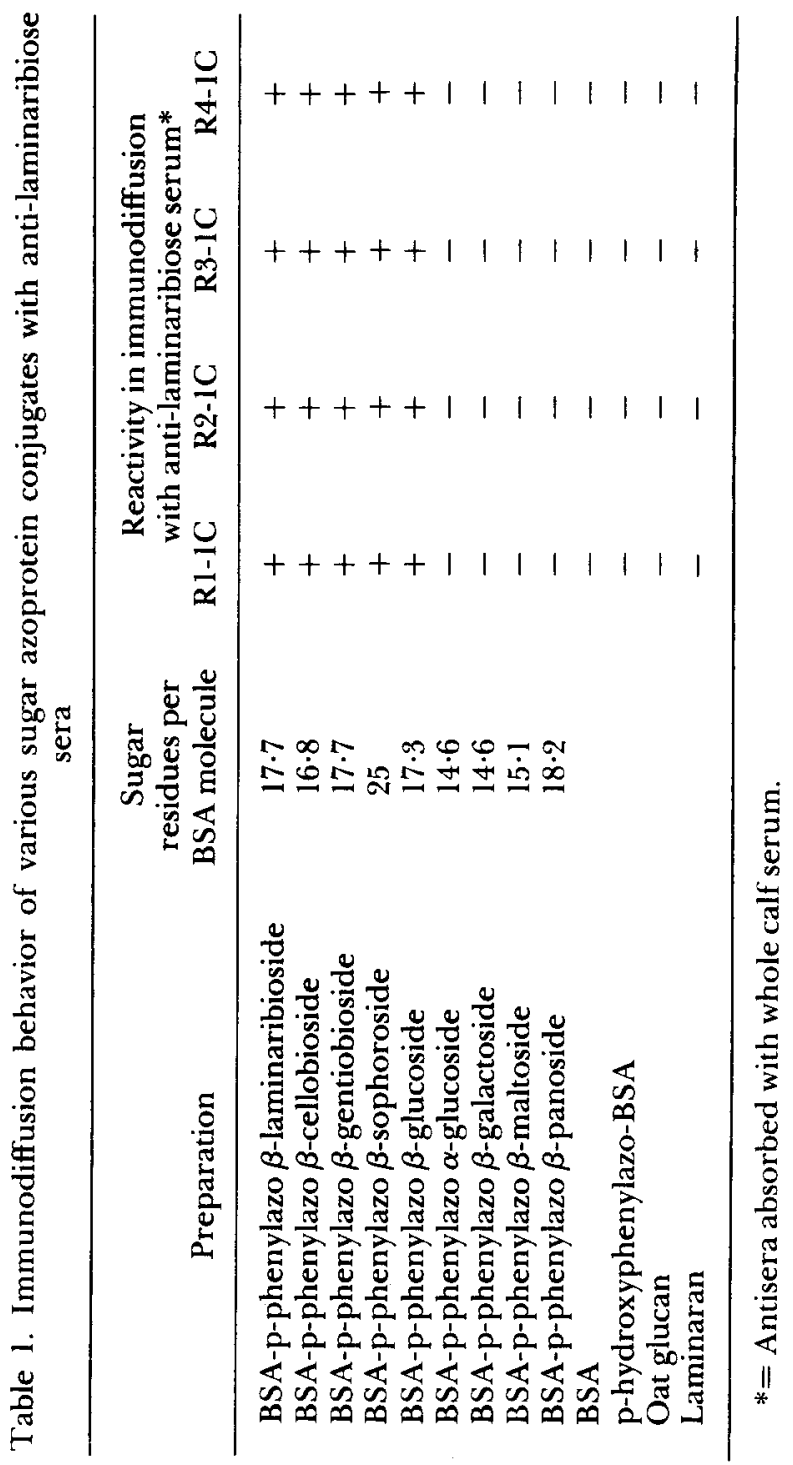


subcutaneous injections $(0.2 \mathrm{ml})$ into the toe pads and hind quarters. Immune sera obtained from bleedings taken 21 days after the last injection were preserved by adding phenol and merthiolate to a final concentration of 0.25 and $0.01 \%$, respectively. Antisera were rendered specific for the introduced $\beta$-laminaribiosyl-phenylazo group by absorbing with whole bovine serum to remove antibodies to carrier protein. Further absorption of sera with p-hydroxyphenylazo-BSA, as described for antisera to BSA-p-phenylazo $\beta$-cellobioside[13], was not carried out, since carrier-absorbed sera failed to react in immunodiffusion with the p-hydroxyphenylazo-BSA conjugate.

Quantitative precipitin studies. Precipitin curves were obtained with $0.5 \mathrm{ml}$ aliquots of rabbit anticonjugate by the procedure described by Kabat and Mayer[27]. Antibody nitrogen $(\mathrm{AbN})$ precipitable by the homologous $\beta$ laminaribiosyl-BSA conjugate and cross-reacting $\beta$-cellobiosyl, $\beta$-sophorosyl and $\beta$-gentiobiosyl-BSA conjugates was determined by micro-Kjeldahl analysis. Precipitin reactions were carried out in a total volume of $1.5 \mathrm{ml}$. Supernatant fluids ( $1.0 \mathrm{ml}$ aliquots) from precipitin reaction mixtures with antiserum $\mathrm{R} 4-1 \mathrm{C}$ at equivalence were assayed for their $\mathrm{Ab} N$ precipitable by various heterologous conjugates.

Quantitative hapten inhibition. Haptens were assayed for their ability to inhibit precipitation of anti-laminaribiose from $50 \mu \mathrm{l}$ of carrier-absorbed antisera R4-1C and R3-1C by $1 \cdot 2 \mu \mathrm{g} \beta$-laminaribiosyl-BSA nitrogen, employing the scaled-down Folin-Ciocalteu method described by Kabat and Schiffman [28]. Hapten inhibition of cross reactions was carried out, employing a test system of $100 \mu \mathrm{l}$ serum R3-1C and 2.0 $\mu \mathrm{g} \beta$-cellobiosyl-BSA nitrogen or $200 \mu \mathrm{l}$ serum R3-1C and $1.5 \mu \mathrm{g}$ $\beta$-sophorosyl-BSA nitrogen. Volumes of serum were chosen so that approximately $7 \mu \mathrm{g} \mathrm{Ab} \mathrm{N}$ was precipitated by an equivalent amount of antigen in the assay system. Hapten inhibitors were added to antiserum and mixtures incubated at $37^{\circ} \mathrm{C}$ for $30 \mathrm{~min}$ before addition of antigen. The total volume of homologous reaction mixtures was adjusted to $0.4 \mathrm{ml}$, while cross reactions employing the $\beta$-cellobiosyl and $\beta$-sophorosyl-BSA conjugates as antigen were carried out in a $0.5 \mathrm{ml}$ volume. Tubes were placed in an ice bath at $0^{\circ}$, kept in a refrigerator for 7 days and mixed twice daily.

Haptens. Glucose was obtained from J. T. Baker and Co. Methyl $\beta$-D-glucopyranoside and p-nitrophenyl $\beta$-D-glucopyranoside were obtained from California Corp. for Biochemical Research. Cellobiose $(\mathrm{Gp} \stackrel{\beta}{\rightarrow} 4 \mathrm{Gp}$ ) was purchased from Pfanstiehl Laboratories. Gentiobiose (Gp $1 \stackrel{\not}{\rightarrow} 6 \mathrm{Gp})$ was prepared by enzymic synthesis as described by Goldstein and Whelan [29] and purified by paper chromatography. The preparation and characterization of sophorose $\left(\mathrm{Gp}^{\stackrel{\beta}{\rightarrow}} 2 \mathrm{Gp}\right.$ ), p-aminophenyl $\beta$-sophoroside and p-nitrophenyl $\beta$-laminaribioside have been previously described [26, 30]. Laminaribiose $(\mathrm{Gp} 1 \stackrel{\beta}{\rightarrow} 3 \mathrm{Gp}$ ) used for hapten inhibition was isolated from pachyman by partial acid hydrolysis, as described by Whelan[31]. Laminaritriose (Gp $1 \stackrel{\beta}{\rightarrow} 3 \mathrm{Gp}$ $1 \stackrel{\beta}{\rightarrow} 3 \mathrm{Gp})$, laminaritetraose $(\mathrm{Gp} \stackrel{\beta}{\rightarrow} 3 \mathrm{Gp} 1 \stackrel{\beta}{\rightarrow} 3 \mathrm{Gp} 1 \stackrel{\beta}{\rightarrow} 3 \mathrm{Gp})$ and an additional sample of laminaribiose were obtained from Dr. A. S. Perlin.

$O$ - $\beta$-D-glucopyranosyl-( $1 \rightarrow 4)$ - $O$ - $\beta$-D-glucopyranosyl $(1 \rightarrow 3)$-D-glucopyranose $(\mathrm{Gp} 1 \stackrel{\beta}{\rightarrow} 4 \mathrm{Gp} 1 \stackrel{\beta}{\rightarrow} 3 \mathrm{Gp}), O-\beta$-D-glucopyranosyl- $(1 \rightarrow 3)-O-\beta$-D-glucopyranosyl$(1 \rightarrow 4)$-D-glucopyranose $(\mathrm{Gp} 1 \stackrel{\beta}{\rightarrow} 3 \mathrm{Gp} \mathrm{l} \stackrel{\beta}{\rightarrow} 4 \mathrm{Gp}), O-\beta$-D-glucopyranosyl-( $1 \rightarrow 3$ )- 
$O$ - $\beta$-D-glucopyranosyl-( $\rightarrow 4$ )-O- $\beta$-D-glucopyranosyl-(1 $\rightarrow 4$ )-D-glucopyranose $(\mathrm{Gpl} \stackrel{\stackrel{B}{\rightarrow}}{3} 3 \mathrm{Gp} 1 \stackrel{\beta}{\rightarrow} 4 \mathrm{Gp} \mathrm{l} \stackrel{\beta}{\rightarrow} 4 \mathrm{Gp}$ ), and $O-\beta$-D-glucopyranosyl-(1 $\rightarrow 4)$-D-glucopyranosyl-( $(\rightarrow 4)$ - $O$ - $\beta$-D-glucopyranosyl-( $(\rightarrow 3)$-D-glucopyranose $(\mathrm{Gp} \mathrm{l} \rightarrow 4 \mathrm{Gp}$ ${ }_{1} \stackrel{\beta}{\rightarrow} 4 \mathrm{Gpl} \stackrel{\beta}{\rightarrow} 3 \mathrm{Gp}$ ) were prepared by selective enzymolysis of oat glucan and lichenin $[32,33]$ and generously provided by Dr. A. S. Perlin. The preparation, purification and properties of these compounds have been described in detail [32-35]. A preparation of 3-O- $\beta$-D-glucopyranosyl-D-galactose $(\mathrm{Gp} 1 \stackrel{\beta}{\rightarrow} 3 \mathrm{Galp})$ [36] was a gift from Dr. H. M. Flowers.

Immunoelectrophoresis and immunodiffusion analyses. Immunoelectrophoresis was carried out essentially as described by Grabar and Burtin [37]. Double diffusion in two dimensions was carried out by a modified Ouchterlony method [27]. Goat anti-rabbit serum used in immunoelectrophoresis was purchased from Hyland Laboratories.

\section{RESULTS}

Immunodiffusion and immunoelectrophoresis with anti-laminaribiose sera. Antisera obtained from four rabbits after a single course of immunization with $\beta$ laminaribiosyl-BSA were examined by immunodiffusion, before and after absorption with whole calf serum. Each antiserum gave an intense band of precipitation with protein carrier (BSA) which disappeared after absorption with whole calf serum.

Absorbed anti-laminaribiose sera (R1-1C, R2-1C, R3-1C, R4-1C) were identical in their immunodiffusion reactions when tested against various sugar-azoprotein conjugates (Table 1). A band of precipitation was obtained with the p-phenylazo-BSA conjugates of $\beta$-laminaribiose, $\beta$-cellobiose, $\beta$-gentiobiose, $\beta$-sophorose and $\beta$-glucose, while conjugates possessing $\alpha$-glucosyl, $\beta$-maltosyl or $\beta$-panosyl groupings failed to give any detectable band..As shown in Fig. 1, the band given by $\beta$-laminaribiosyl-BSA spurs over bands obtained with $\beta$ cellobiosyl, $\beta$-sophorosyl and $\beta$-glucosyl-BSA conjugates. While bands given by the $\beta$-glucosyl and $\beta$-sophorosyl conjugates show complete fusion with one another, the band obtained with $\beta$-cellobiosyl-BSA spurs over that given by the $\beta$-sophorosyl conjugate. Although not shown in Fig. 1, $\beta$-gentiobiosyl-BSA showed the same immunodiffusion behavior as $\beta$-glucosyl-BSA. When allowed to stand for long periods of time or when antigen was employed at high concentrations, several of the bands in immunodiffusion showed distinct splitting, as seen in Fig. 1, similar to that reported for the cellobiose-anticellobiose system [13].

Reaction of antiserum R4-1C with an equivalent amount of $\beta$-laminaribiosylBSA removes all the antibody present, yielding a supernatant which no longer gives a band in immunodiffusion with any sugar-azoprotein conjugate listed in Table 1 . While absorption with $\beta$-cellobiosyl-BSA eliminates bands given by $\beta$-gentiosiosyl, $\beta$-glucosyl and $\beta$-sophorosyl conjugates, the band obtained with $\beta$-laminaribiosyl-BSA persists.

Oat glucan and laminaran, naturally occurring polysaccharides possessing $\beta$ - $(1 \rightarrow 3)$-linked glucose units, failed to give a band with anti-laminaribiose sera.

Immunoelectrophoresis of rabbit anti-laminaribiose was carried out on absorbed sera employing the $\beta$-laminaribiosyl-BSA conjugate to localize specific antibody and a goat anti-rabbit reagent to demonstrate rabbit serum proteins. 
As can be seen in Fig. 2, a single arc of precipitation is given by conjugate in the $\gamma_{2}$-globulin region with each of the four sera tested. Immunoelectrophoretic evidence of an $\operatorname{lgM}$ or $\operatorname{lgA}$ class of anti-laminaribiose was not seen.

Quantitative precipitin determinations and supernatant analyses. Quantitative precipitin curves obtained with four carrier-absorbed, anti-laminaribiose sera employing $\beta$-laminaribiosyl-BSA as antigen are shown in Fig. 3. The maximum amount of $\mathrm{Ab} \mathrm{N}$ precipitated from $0.5 \mathrm{ml}$ anticonjugate by homologous antigen varied with individual rabbit sera and was found to be $76,100,115$ and $128 \mu \mathrm{g}$ Ab N for sera R1, R2, R3 and R4, respectively.

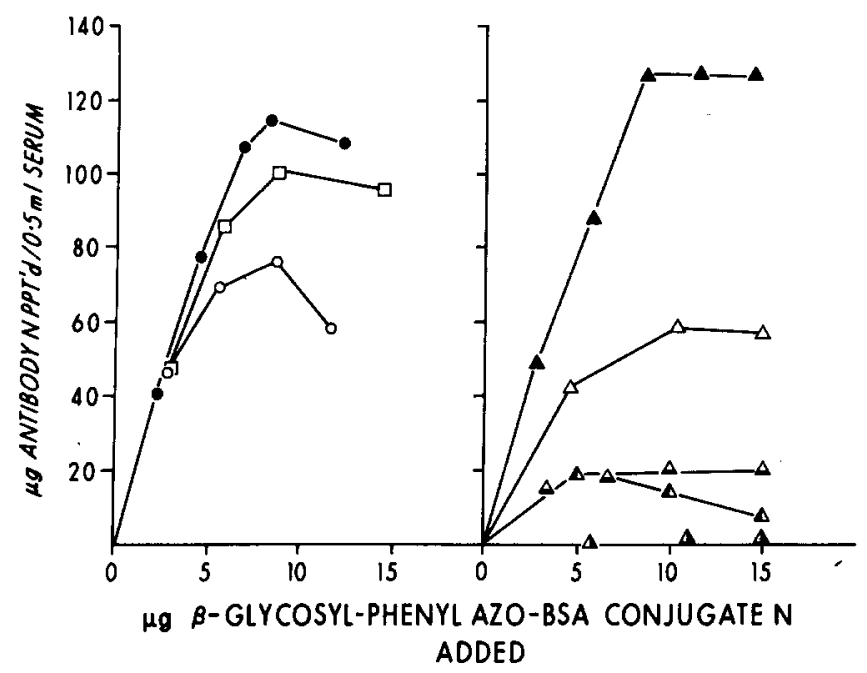

Fig. 3. Quantitative precipitin curves obtained with carrier absorbed rabbit anti-laminaribioside. Antibody $\mathrm{N}$ precipitated by $\beta$-laminaribiosyl-BSA from serum R1-1C, O; R2-1C, 9 ; R3-1C, $\square$. Antibody N precipitated from serum R4-1C by $\beta$-laminaribiosyl-BSA, $\Delta ; \beta$-cellobiosyl-BSA, $\triangle ; \beta$-sophorosyl-BSA, $\Delta ; \beta$-gentiobiosyl-BSA, $\Delta$ and $\beta$-glucosyl-BSA, $\Lambda$.

Cross reactions of serum $\mathrm{R} 4-1 \mathrm{C}$ observed in immunodiffusion (Table 1 and Fig. 1) with conjugates possessing the $\beta$-linked glucosyl unit were examined by the quantitative precipitin method. As shown in Fig. 3, the BSA conjugates of $\beta$-gentiobiose and $\beta$-sophorose each removed $19 \mu \mathrm{g}$ from $0.5 \mathrm{ml}$ serum, while $\beta$-cellobiosyl-BSA removed $58 \mu \mathrm{g}$ Ab N. Although the $\beta$-glucosyl-BSA conjugate gave a faint, detectable band in immunodiffusion, it failed to give a measurable amount of precipitate in the quantitative precipitin method with the amounts of serum employed.

Supernatant fluids obtained at equivalence from precipitin reactions of antiserum $\mathrm{R} 4-1 \mathrm{C}$ shown in Fig. 3 were assayed for residual $\mathrm{Ab} \mathrm{N}$ recoverable by addition of heterologous conjugate. In agreement with immunodiffusion findings, reaction with homologous antigen $\beta$-laminaribiosyl-BSA gives a supernatant fluid from which no additional antibody nitrogen could be recovered by addition of $\beta$-cellobiosyl, $\beta$-sophorosyl, $\beta$-gentiobiosyl or $\beta$-glucosyl-BSA conjugates. Supernatant fluids from the cross reaction of serum R4-1C with 


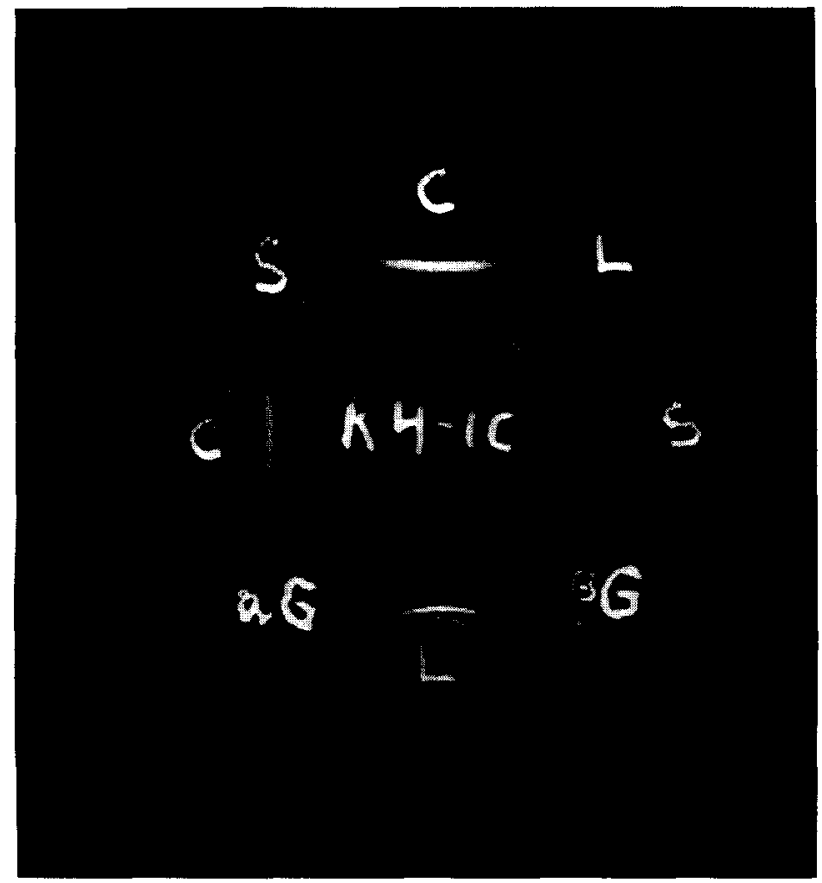

Fig. 1. Immunodiffusion with anti-laminaribioside serum R4-1C absorbed with carrier protein. Peripheral wells labelled L contain $\beta$-laminaribiosyl-BSA, wells labelled $\mathrm{C}, \mathrm{S}, \alpha-\mathrm{G}$ and $\beta$-G contain $\beta$-cellobiosyl, $\beta$-sophorosyl, $\alpha$-glucosyl and $\beta$-glucosyl-BSA conjugates, respectively. 


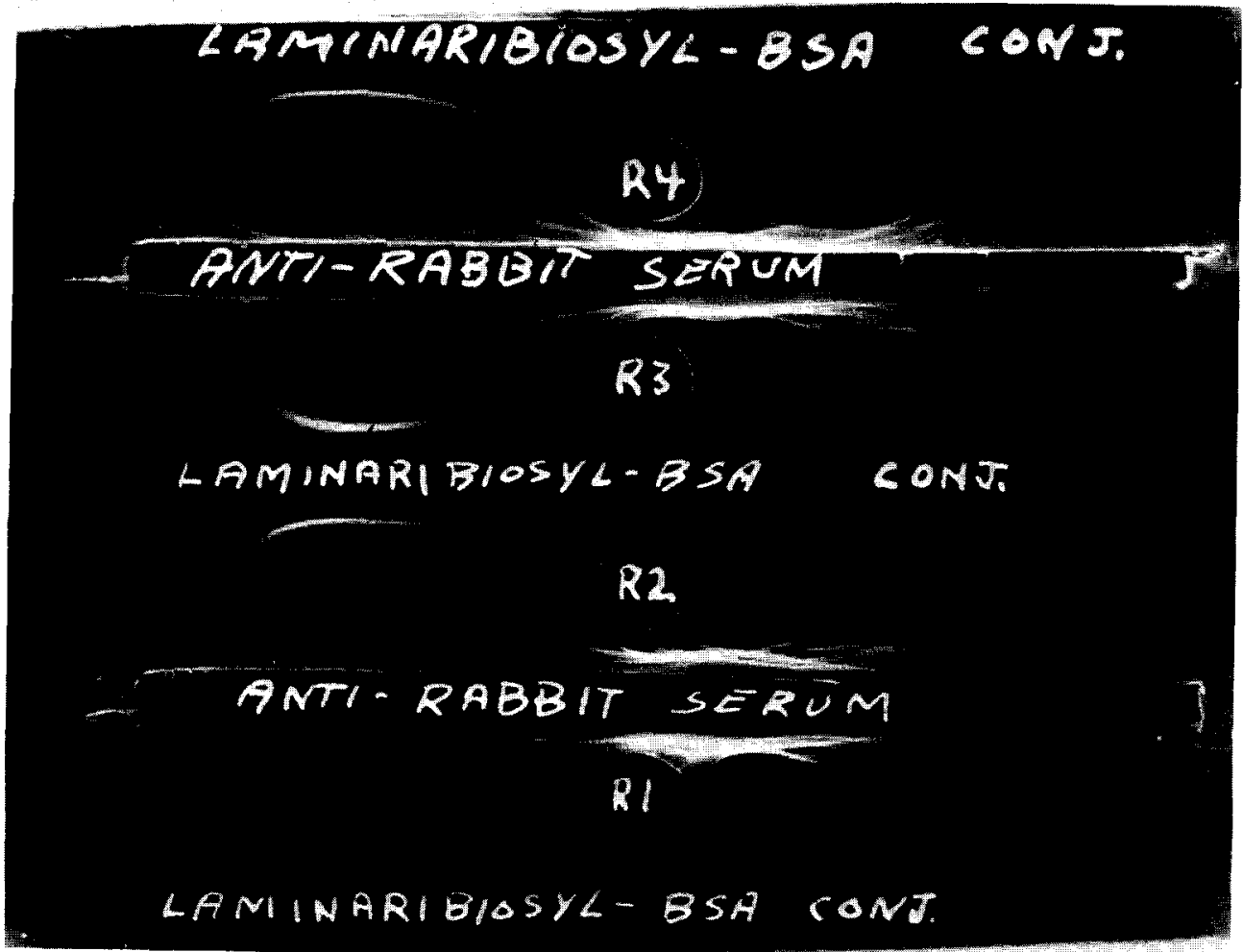

Fig. 2. Immunoelectrophoresis of anti-laminaribioside sera. Central wells labelled R1, R2, R3, R4 contain carrier absorbed, rabbit anti- $\beta$-laminaribiosyl-BSA sera. Troughs labelled laminaribiosyl-BSA contain p-phenylazo- $\beta$-laminaribiosyl-BSA conjugate $(50 \mu \mathrm{g} \mathrm{N} / \mathrm{ml})$, while troughs labelled anti-rabbit serum contain goat anti-whole rabbit serum. 